International Mathematical Forum, Vol. 10, 2015, no. 3, 105 - 109 HIKARI Ltd, www.m-hikari.com http://dx.doi.org/10.12988/imf.2015.412200

\title{
On the Extension of Properties of Cubic Measures
}

\author{
Otanga Levi Olwamba
}

\author{
Department of Mathematics \\ Masinde Muliro University of Science and Technology \\ P.O. Box 536, Kakamega, Kenya
}

Copyright (C) 2014 Otanga Levi Olwamba. This is an open access article distributed under the Creative Commons Attribution License, which permits unrestricted use, distribution, and reproduction in any medium, provided the original work is properly cited.

\begin{abstract}
We consider the application and extension of properties of cubic measure to measurable spaces, measurable and integrable functions. The concepts of finiteness and $\sigma$ - finiteness of a measure function are assumed and invoked whenever appropriate.
\end{abstract}

Mathematics Subject Classification: 28B15, 28B20

Keywords: Finite function, Cubic measure

\section{Introduction}

In the previous results, many scholars studied measurable sets generated by semirings and sigma algebras. Attention was given to developing a measure for one and two dimensional measurable spaces. A measure was not defined for measurable sets in finite-dimensional Euclidean spaces $\Re^{n}$ for $n>2$. In this article, we investigate the extension of properties of cubic measure to measurable spaces, measurable and integrable functions generated by $\sigma$ - rings of subsets of three dimensional spaces, with additional properties involving monotone convergence theorem. For the notations and basic concepts used in the article reference may be made to $[1,2,3,4,6]$ 


\section{The Results}

Proposition 1. Each measurable subset of $X \times Y \times Z$ is contained in some measurable cuboid, that is, if $M \subset X \times Y \times Z$ and $M \in \rho \times \tau \times \psi$, then there exists a measurable cuboid $E \times F \times H$ such that $M \subset E \times F \times H$.

Proof. Each measurable set in $X \times Y \times Z$ is contained in a countable union of sets in $E \times F \times H$, where $E \subset X, F \subset Y$, and $H \subset Z$. Let $M \in \rho \times \tau \times \psi$. There exists a sequence of measurable cuboids $\left(E_{n} \times F_{n} \times H_{n}\right)_{n=1}^{\infty}$ such that $M \subset \cup_{1}^{\infty} E_{n} \times F_{n} \times H_{n}$. Defining $E=\cup_{1}^{\infty} E_{n}, F=\cup_{1}^{\infty} F_{n}$ and $H=\cup_{1}^{\infty} H_{n}$, we have $M \subset \cup_{1}^{\infty} E_{n} \times F_{n} \times H_{n}=E \times F \times H \Rightarrow M \subset E \times F \times H$

Proposition 2. Let $\Upsilon$ be the class of measurable subsets of $X$ such that $G(\Upsilon)=\rho$, $\Phi$ be the class of measurable subsets of $Y$ such that $G(\Phi)=\tau$, and $\Omega$ be the class of measurable subsets of $Z$ such that $G(\Omega)=\psi$. If $\Delta=$ $\{E \times F \times H: E \in \Upsilon, F \in \Phi$ and $H \in \Omega\}$, then $G(\Delta)=\rho \times \tau \times \psi$.

Proof. Since $\Delta$ is measurable, it implies that $\Delta \in \rho \times \tau \times \psi$. Given that $G(\Delta)$ is the smallest $\sigma-$ ring generated by $\Delta$, it follows that $G(\Delta)$ is a subset of a $\sigma-\operatorname{ring} \rho \times \tau \times \psi$ generated by subsets of $X \times Y \times Z$. Fix $E \in \Upsilon$ and $F \in \Phi$. Consider the class defined by $A=\{P \subset Z: E \times F \times P \in G(\Delta\}$. Since $E \times F \times\left(\cup_{1}^{\infty} P_{n}\right)=\cup_{1}^{\infty} E \times F \times P_{n} \in A$ and $E \times F \times\left(P_{1}-P_{2}\right)=$ $\left\{E \times F \times P_{1}-E \times F \times P_{2}\right\} \in A$, it follows that A is closed under countable unions and differences. Therefore, A is $\sigma-$ ring. Since A contains the class $\Omega$ of measurable subsets of $\mathrm{Z}$ and $G(\Omega)=\psi$ is the smallest $\sigma$-ring generated by $\Omega$, we have $G(\Omega)=\psi \subset A$. Therefore if $E \in \Upsilon, F \in \Phi$ and $H \in \Omega$, then $E \times F \times H \in G(\Delta)$. Fix $E \in \Upsilon$ and $H \in \Omega$. Define $B=\{T \subset Y$ : $E \times T \times H \in G(\Delta)\}$. Since $E \times\left(\cup_{1}^{\infty} T_{n}\right) \times H=\cup_{1}^{\infty} E \times T_{n} \times H \in B$ and $E \times\left(T_{1}-T_{2}\right) \times H=E \times T_{1} \times H-E \times T_{2} \times H \in B$, it follows by definition that B is a $\sigma-$ ring. Since $\mathrm{B}$ contains the class $\Phi$ of measurable subsets of $\mathrm{Y}$, we have $G(\Phi)=\tau \subset B$. Therefore, if $E \in \Upsilon, F \in \Phi$ and $H \in \Omega$, then $E \times F \times H \in G(\Delta)$. Finally, fix $F \in \Phi$ and $H \in \Omega$. Suppose $C=\{S \subset X: S \times F \times H \in G(\Delta)\}$. Since $\left(\cup_{1}^{\infty} S_{n}\right) \times F \times H \in C$ and $\left(S_{1}-S_{2}\right) \times F \times H=S_{1} \times F \times H-S_{2} \times F \times H \in C$, it implies that $\mathrm{C}$ is a $\sigma-$ ring. Since $\mathrm{C}$ contains the class $\Upsilon$ of subsets of $\mathrm{X}$, then $G(\Upsilon)=\rho \subset C$. If $E \in \Upsilon, F \in \Phi$ and $H \in \Omega$, then $E \times F \times H \in G(\Delta)$. We therefore conclude that $\rho \times \tau \times \psi \subset G(\Delta)$. Since $G(\Delta) \subset \rho \times \tau \times \psi$, we have $G(\Delta)=\rho \times \tau \times \psi$

Proposition 3. If $f: Y \times Z \rightarrow X \times Y \times Z$ is a function defined on $Y \times Z$ and $\rho \times \tau \times \psi$ is $\sigma-$ ring of subsets of $X \times Y \times Z$, then the class of all subsets of the form $f^{-1}(M)$ where $M$ is in $\rho \times \tau \times \psi$, is a $\sigma$-ring of subsets of $Y \times Z$.

Proof. Rectangles are used to generate $\sigma$-rings of subsets of $X \times Y$. Based on these findings, we generate $\sigma$-rings of subsets $E \times F \times H$ of $X \times Y \times Z$ 
and show that the inverse image of the $\sigma-$ ring of subsets of $X \times Y \times Z$ is a $\sigma$-ring of subsets of $Y \times Z$.Let $M_{1}$ and $M_{2}$ be both in $\rho \times \tau \times \psi$ and $\mathrm{K}$ be the class of subsets of $Y \times Z$. Since $\rho \times \tau \times \psi$ is a $\sigma-$ ring, it is closed under set differences and countable unions.If $M_{1}-M_{2} \in \rho \times \tau \times \psi$, then $f^{-1}\left(M_{1}-M_{2}\right)=$ $f^{-1}\left(M_{1}\right)-f^{-1}\left(M_{2}\right)$. But $f^{-1}\left(M_{1}\right)-f^{-1}\left(M_{2}\right) \in K \subset Y \times Z$.Therefore, $\mathrm{K}$ is closed under set differences.Let $\left(E_{i} \times F_{i} \times H_{i}\right)_{1}^{\infty}$ be a sequence of subsets in $\rho \times \tau \times \psi$. Since $\rho \times \tau \times \psi$ is a $\sigma-$ ring, then $\cup_{1}^{\infty} E_{i} \times F_{i} \times H_{i} \in \rho \times \tau \times \psi$.Therefore, $f^{-1}\left(\cup_{1}^{\infty} E_{i} \times F_{i} \times H_{i}\right)=\cup_{1}^{\infty} f^{-1}\left(E_{i} \times F_{i} \times H_{i}\right) \in K \subset Y \times Z$.From these facts, it is clear that $\mathrm{K}$ is closed under countable unions. Hence, $\mathrm{K}$ is a $\sigma$-ring of subsets of $Y \times Z$.

Proposition 4. If $h$ is a measurable function with respect to $\rho \times \tau \times \psi$, then for $x \in X$, the function $h_{x}$ is measurable with respect to $\tau \times \psi$.

Proof. Fix $x \in X$ and let $N\left(h_{x}\right)=(N(h))_{x}$.Now, $N\left(h_{x}\right)=\{(y, z) \in$ $Y \times Z: h(x, y, z) \neq 0\}$. If $(y, z) \in N\left(h_{x}\right), \Leftrightarrow h_{x}(y, z) \neq 0 \Leftrightarrow h(x, y, z) \neq 0$ $\Leftrightarrow(x, y, z) \in N(h) \Leftrightarrow(y, z) \in(N(h))_{x} \Rightarrow N\left(h_{x}\right)=(N(h))_{x}$.If B is a Borel set of real numbers, then $h_{x}^{-1}(B)=\left(h^{-1}(B)\right)_{x}$. The following relations are equivalent.Suppose $(y, z) \in h_{x}^{-1}(B) \Leftrightarrow h_{x}(y, z) \in h_{x}\left(h_{x}^{-1}(B)\right) \Leftrightarrow h(x, y, z) \in B$ $\Leftrightarrow h^{-1}(h(x, y, z)) \in h^{-1}(B) \Leftrightarrow(x, y, z) \in h^{-1}(B) \Leftrightarrow(y, z) \in\left(h^{-1}(B)\right)_{x} \Rightarrow$ $h_{x}^{-1}(B)=\left(h^{-1}(B)\right)_{x}$. Since $N(h) \cap h^{-1}(B)$ is by definition measurable, the set $\left(N(h) \cap h^{-1}(B)\right)_{x}=(N(h))_{x} \cap\left(h^{-1}(B)_{x}=N\left(h_{x}\right) \cap h_{x}^{-1}(B)\right.$ is measurable. Therefore, $h_{x} \in \tau \times \psi$.

Proposition 5. If $(X, \rho, \mu),(Y, \tau, v)$ and $(Z, \psi, \omega)$ are finite measure spaces, then for every measurable set $M$ in $X \times Y \times Z$, the functions $f_{M}, g^{M}$ and $h^{M}$ are integrable and $\int f_{M} \delta_{\mu}=\int g^{M} \delta_{v}=\int h^{M} \delta_{\omega}$.

Proof. Since $\mathrm{M}$ is a set in $\rho \times \tau \times \psi$, the functions $f_{M}, g^{M}$ and $h^{M}$ are bounded. Also the functions $f_{M}, g^{M}$ and $h^{M}$ are measurable. Since $(X, \rho, \mu)$, $(Y, v, \tau)$ and $(Z, \psi, \omega)$ are finite measure spaces and the functions $f_{M}, g^{M}$ and $h^{M}$ are bounded and measurable, they are integrable. Let $E \times F \times H$ be any measurable cuboid, such that $\mu(E)<\infty, v(F)<\infty$ and $\omega(H)<\infty$. For finite measure spaces $(X, \rho, \mu),(Y, \tau, v)$ and $(Z, \psi, \omega)$ and integrable functions $\mathrm{f}, \mathrm{g}$ and $\mathrm{h}$, it is established that $\int f_{E \times F \times H} \delta_{\mu}=\int g^{E \times F \times H} \delta_{v}=\int h_{E \times F \times H} \delta_{\omega}$ .If $M=E \times F \times H$, then $\int f_{M} \delta_{\mu}=\int g^{M} \delta_{v}=\int h^{M} \delta_{\omega}$.If M belongs to the ring $\mathrm{R}$ generated by $E \times F \times H$, then $\mathrm{M}$ can be written as a finite union of measurable cuboids. Therefore M satisfies $\int f_{M} \delta_{\mu}=\int g^{M} \delta_{v}=\int h^{M} \delta_{\omega}$. Let us define real valued set functions $\iota, \kappa$ and $\lambda$ on $\rho \times \tau \times \psi$ by $\iota(M)=$ $\int f_{M} \delta_{\mu} \kappa(M)=\int g^{M} \delta_{v} \lambda(M)=\int h^{M} \delta_{\omega}$. Now $\iota=\kappa=\lambda$ on R. We need to show that $\iota=\kappa=\lambda$ on a $\sigma-\operatorname{ring} \mathrm{G}(\mathrm{R})$ generated by R.This implies that $\iota, \kappa$ and $\lambda$ are (finite) measures on $\rho \times \tau \times \psi$. A measure $\lambda$ defined on a measurable space $(X \times Y \times Z, \rho \times \tau \times \psi)$ is said to be finite if it obeys the 
properties of positivity, additivity, monotonicity, continuity and $\lambda(M)<\infty$ for a measurable subset $\mathrm{M}$ of $X \times Y \times Z$.If $\mathrm{M}$ and $\mathrm{N}$ are disjoint measurable sets with respect to $\rho \times \tau \times \psi$, then $f_{M \cup N}=f_{M}+f_{N}$. Integrating term by term with respect to $\mu$, we obtain $\int f_{M \cup N} \delta_{\mu}=\int f_{M} \delta_{\mu}+\int f_{N} \delta_{\mu}$. But $\iota(M)=$ $\int f_{M} \delta_{\mu}, \iota(N)=\int f_{N} \delta_{\mu}$ and $\iota(M \cup N)=\int f_{M \cup N} \delta_{\mu}$. By the additive property of the measure function $\iota$ becomes $\iota(M \cup N)=\iota(M)+\iota(N)$. Following the same procedure, we conclude that the set functions $\kappa$ and $\lambda$ are additive.The set function $\iota$ is monotone. That is, if $\mathrm{M}$ and $\mathrm{N}$ are sets in $\rho \times \tau \times \psi$ such that $M \subset N$, then $f_{M} \leq f_{N} \Rightarrow \int f_{M} \delta_{\mu} \leq \int f_{N} \delta_{\mu} \Rightarrow \iota(M) \leq \iota(N)$. If $\mathrm{M}$ is a null set i.e $M=\phi$, then $\iota(M)=\iota(\phi)=0$. Let $M_{n}$ be a sequence of sets in $\rho \times \tau \times \psi$ such that $M_{n} \uparrow M$, then $f_{M_{n}} \uparrow f_{M}$. By monotone convergence theorem, we have $\int f_{M_{n}} \delta_{\mu} \uparrow \int f_{M} \delta_{\mu} \Rightarrow \iota\left(M_{n}\right) \uparrow \iota(M)$. Therefore, we conclude that $\iota$ is a finite measure. From the same reasoning, the set functions $\kappa$ and $\lambda$ are finite measures. Since $\iota=\kappa=\lambda$ on $\mathrm{R}$, it follows from unique extension theorem that $\iota=\kappa=\lambda$ on $\sigma$-ring $\mathrm{G}(\mathrm{R})$. Hence $\iota(M)=\kappa(M)=\lambda(M)$. $\Rightarrow$ $\int f_{M} \delta_{\mu}=\int g^{M} \delta_{v}=\int h^{M} \delta_{\omega}$.

Proposition 6. If $(X, \rho, \mu),(Y, \tau, v)$ and $(Z, \psi, \omega)$ are finite measure spaces, there exists a unique measure $\lambda$ on $\rho \times \tau \times \psi$ such that $\lambda(E \times F \times H)=$ $\mu(E) v(F) \omega(H)$ for every measurable cuboid $E \times F \times H$. Moreover $\lambda$ is finite and $\lambda(M)=\int f_{M} \delta_{\mu}=\int g^{M} \delta_{v}=\int h^{M} \delta_{\omega}$ for every measurable set $M$ in $X \times Y \times Z$.

Proof. Let us define a set function $\lambda$ on $\rho \times \tau \times \psi$ by the formula $\lambda(M)=$ $\int f_{M} \delta_{\mu}$. Now $\lambda$ is a finite measure on $\rho \times \tau \times \psi$ and $\lambda(M)=\int f_{M} \delta_{\mu}=\int g^{M} \delta_{v}=$ $\int h^{M} \delta_{\omega}$.If $E \times F \times H$ is a measurable cuboid, then $\int f_{E \times F \times H} \delta_{\mu}=\int g^{E \times F \times H} \delta_{v}=$ $\int h^{E \times F \times H} \delta_{\omega}=\mu(E) v(F) \omega(H)$.If $M=E \times F \times H$, then $\lambda(M)=\lambda(E \times F \times$ $H)=\int f_{M} \delta_{\mu}=\int g^{M} \delta_{v}=\int h^{M} \delta_{\omega}$.If $E \times F \times H$ is a measurable cuboid, then $\lambda(E \times F \times H)=\mu(E) v(F) \omega(H)$. Suppose $\kappa$ is any measure on $\rho \times \tau \times \psi$ such that $\kappa(E \times F \times H)=\mu(E) v(F) \omega(H)$ for every measurable cuboid $E \times F \times H$.It follows that $\lambda=\kappa$ on the ring $\mathrm{R}$ generated by the measurable cuboids.By unique extension theorem, we have $\lambda=\kappa$ on $\mathrm{G}(\mathrm{R})$. Suppose $\lambda=\mu \times v \times \omega$ is a finite measure on $\rho \times \tau \times \psi$, then $(\mu \times v \times \omega)(E \times F \times H)=\mu(E) v(F) \omega(H)$ for every measurable cuboid $E \times F \times H$.

\section{References}

[1] Charalambos, D. A and Owen, B(1989), Principles of Real Analysis ( $2^{\text {nd }}$ Edition), Department of Mathematical Sciences, Indiana University at Indianapolis, Indiana.

[2] Folland G.B. (1999), Real Analysis, Modern Techniques and Their Applications ( $2^{\text {nd }}$ Edition), Wiley and Sons. 
[3] Pantsulaia, G. (2010), Standard Product on Arbitrary Family of Sigma-finite Borel Measures, Vol. 16, pp. 84-93, Springer.

[4] Royden, H.L. (1988), Real Analysis ( $3^{\text {rd }}$ Edition), Macmillan Publishing Company, USA.

[5] Sergey, G. B (2009), Isoperimetric Constants for Product Measures, Journal of Mathematical Sciences, Vol. 159, No.1, pp. 47-53. http://dx.doi.org/10.1007/s10958-009-9425-z

[6] Torence, T (2011), An Introduction to Measure Theory, Department of Mathematics, UCLA, Los Angeles, pp. 179-208.

Received: December 19, 2014; Published: February 12, 2015 